\title{
Long-term results of concurrent chemoradiotherapy for T3/T4 locally advanced nasopharyngeal carcinoma
}

\author{
CAN XIAO $^{1 *}$, LILI WANG $^{2 *}$, YANG JIAO ${ }^{3}$, KEKANG SUN $^{3}$, SONGBING $_{\text {QIN }}^{2}$, \\ XIAOTING XU ${ }^{2}$, JIAN GUO ${ }^{2}$ and JUYING ZHOU ${ }^{2}$ \\ Departments of ${ }^{1}$ Stomatology and ${ }^{2}$ Radiation Oncology, The First Affiliated Hospital of Soochow University, \\ Soochow University, Suzhou, Jiangsu 215006; ${ }^{3}$ School of Radiation Medicine and Protection, Medical \\ College of Soochow University, Soochow University, Suzhou, Jiangsu 215123, P.R. China
}

Received November 5, 2012; Accepted January 24, 2013

DOI: $110.3892 / \mathrm{mco} .2013 .75$

\begin{abstract}
Nasopharyngeal carcinoma (NPC) is one of the most commonly diagnosed head and neck malignancies. This study investigated the outcome of locally advanced NPC patients on concurrent intensity-modulated radiation therapy (IMRT) and chemotherapy with docetaxel, cisplatin and 5-fluorouracil (TPF). A total of 226 patients with locally advanced NPC received IMRT, with a total dose of 65-70 Gy and concurrent chemotherapy, with 2 cycles of TPF administered during radiotherapy, between March, 2005 and March, 2007. An additional 2 to 4 cycles of chemotherapy were administered every 21 days following radiotherapy. With a median follow-up time of 35 months (range, 7-60), the 5-year overall survival (OS) rate was $81.4 \%$, with 93.6 and $75.0 \%$ for T3 and T4 lesions, respectively, $(\mathrm{P}=0.001)$. The 5-year progression-free survival (PFS) was $50.4 \%$, with 66.7 and $46.9 \%$ for T3 and T4 lesions, respectively $(\mathrm{P}<0.001)$. T-classification was a significant prognostic factor for PFS and OS. The subgroup analysis revealed that pterygopalatine fossa invasion was associated with a significantly lower 5-year PFS ( $\mathrm{P}=0.001)$ and OS $(\mathrm{P}=0.002)$, foramen rotundum invasion was associated with a significantly lower 5-year PFS $(\mathrm{P}<0.001)$ and $\mathrm{OS}(\mathrm{P}=0.004)$, foramen ovale invasion was associated with a significantly lower 5-year PFS $(\mathrm{P}=0.013)$ and $\mathrm{OS}(\mathrm{P}=0.024)$ and foramen lacerum and cavernous sinus invasion were associated with a significantly lower 5-year PFS
\end{abstract}

Correspondence to: Professor JuYing Zhou, Department of Radiation Oncology, The First Affiliated Hospital of Soochow University, 188 Shizi Street, Suzhou, Jiangsu 215006, P.R. China

E-mail: zhjuying@sohu.com

Abbreviations: NPC, nasopharyngeal carcinoma; IMRT, intensity-modulated radiation therapy; OS, overall survival; PFS, progression-free survival; OAR, organs at risk; CT, computed tomography; GTV, gross tumor volume; CTV, clinical tumor volume; RTOG, radiation therapy oncology group

*Contributed equally

Key words: nasopharyngeal carcinoma, intensity-modulated radiation therapy, chemotherapy
$(\mathrm{P}<0.001$ and $\mathrm{P}<0.001$, respectively). Concurrent chemoradiotherapy is an advocated regimen for patients with locally advanced NPC, since it exhibits satisfactory 5-year PFS and OS rates. Our results suggest that the estimation of invasive range may identify a subgroup of patients with a higher risk of locoregional failure who may be better candidates for this treatment strategy.

\section{Introduction}

Nasopharyngeal carcinoma (NPC) is the most commonly diagnosed head and neck malignancy in Southeast Asia and $70 \%$ of patients present with locally advanced-stage cancer at the time of diagnosis (1-3). Moreover, NPC demonstrates the highest incidence rates of distant metastasis among all head and neck cancers. Due to anatomical restrictions, the standard treatment for NPC is definitive radiotherapy. The benefits of chemotherapy administered concurrently with radiation are supported by the present data, demonstrating significant increases in progression-free survival (PFS), as well as overall survival (OS) $(4,5)$.

Although considered essential for patients with locally advanced NPC, the therapeutic value of chemotherapy administered concurrently with intensity-modulated radiation therapy (IMRT) and the optimal strategy of combining the two, has not yet been sufficiently addressed. The rationale of concurrent chemotherapy and IMRT in the treatment of NPC is mostly derived from experience with conventional radiotherapy. Concurrent chemoradiotherapy has shown significant benefits following treatment; however, whether it offers equal therapeutic benefits to all the patient subgroups with locally advanced NPC remains to be determined. The objective of this study was to assess treatment outcomes and elucidate the efficacy of concurrent chemoradiotherapy, by analyzing results obtained from a relatively large group of patients with locally advanced NPC, uniformly treated with IMRT and concurrent chemotherapy.

\section{Materials and methods}

Patients and pretreatment evaluation. A total of 226 patients with histologically diagnosed non-metastatic NPC were treated with concurrent chemoradiotherapy at the First Affiliated Hospital of Soochow University (Suzhou, China), 
between March, 2005 and March, 2007. The pretreatment evaluation consisted of a complete history and physical examination, flexible fiberoptic endoscopic examination, blood chemistry tests, urinalysis, chest X-ray, electrocardiogram, computed tomography (CT) scans of the nasopharynx and neck, magnetic resonance imaging (MRI) scans of the head and neck, bone emission computed tomography scans, liver and abdominal lymph node ultrasounds and dental evaluation. Positron emission tomography scans and CT scans of the chest and abdomen were optional and performed when clinically indicated. Tumors were staged according to the Guangzhou staging system (2008). Patients who presented with evidence of distant metastasis were not eligible for this treatment protocol. The characteristics of the 226 patients are listed in Table I.

IMRT techniques. IMRT was performed using a commercial stereotactic radiotherapy system (Varian Eclipse; Varian Medical Systems, Inc., Palo Alto, CA, USA), in order to deliver revolving conformal radiation based on multileaf collimator to the target, using a 6-MV linear accelerator (Varian 23EX; Varian Medical Systems, Inc.). The patients were immobilized in the supine position with thermoplastic masks. CT planning scans with intravenous contrast material were performed, using 3-mm slices from the head to $2 \mathrm{~cm}$ below the level of the sternoclavicular joints. The primary and nodal gross tumor volumes (GTV-nx) and the cervical lymph node gross tumor volumes (GTV-nd) included the gross diseases visualized on $\mathrm{CT}$ and MRI. The high-risk clinical tumor volume (CTV-1) included GTV and a 5- to 10-mm margin, encompassing the entire nasopharyngeal mucosa, as well as a $5-\mathrm{mm}$ submucosal volume. CTV-2 was designed for potentially involved regions, including the nasopharyngeal cavity, maxillary sinus, pterygopalatine fossa, posterior ethmoid sinus, parapharyngeal space, skull base, anterior third of the clivus and cervical vertebrae, inferior sphenoid sinus and cavernous sinus, as well as the retropharyngeal lymph nodal regions, from the base of skull to the cranial edge of the second cervical vertebra. The planning target volume was created based on each volume, with an additional 2- to 3-mm margin, which allowed for setup variability. Critical normal structures, including brainstem, spinal cord, parotid glands, optic nerves, optic chiasm, lens, eyeballs, temporal lobes, temporomandibular joints, mandible and hypophysis, were contoured and set as organs at risk (OAR) during optimization. The radiation doses prescribed in the protocol were as follows: a total dose of 65-70 Gy in 32 fractions to the GTV-nx and GTV-nd, 56-60 Gy in 32 fractions to the CTV-1 and 50-52 Gy in 28 fractions to the CTV-2. The patients were treated with one fraction daily for five days per week. The dose received by each OAR should be less than its tolerance limit, according to the radiation therapy oncology group (RTOG) 0225 protocol.

Chemotherapy. Concurrent chemotherapy was administered to all patients, in order to prevent disease progression during IMRT treatment planning. Concurrent chemotherapy consisted of 6 cycles of TPF regimen (cisplatin $80-100 \mathrm{mg} / \mathrm{m}^{2}$ i.v. on Day 1, 5-fluorouracil $500-600 \mathrm{mg} / \mathrm{m}^{2}$ i.v. on Days 1-5 and docetaxel $135-175 \mathrm{mg} / \mathrm{m}^{2}$ i.v. on Day 1). The patients received 2 courses of chemotherapy every 28 days during radiotherapy, followed by an additional 2-4 cycles of chemotherapy every 21 days after radiotherapy.
Table I. Patient characteristics in the study population of 226 nasopharyngeal carcinoma patients, according to the Guangzhou staging system (2008).

\begin{tabular}{lrc}
\hline Characteristics & No. & Percentage (\%) \\
\hline Patients & 226 & 100 \\
Age (years) & & \\
$>60$ & 71 & 31.4 \\
$\leq 60$ & 155 & 68.6 \\
Gender & & \\
Male & 154 & 68.1 \\
Female & 72 & 31.9 \\
T-classification & & \\
T3 & 78 & 34.5 \\
T4 & 148 & 65.5 \\
N-classification & & \\
N0 & 36 & 15.9 \\
N1 & 72 & 31.9 \\
N2 & 112 & 49.6 \\
N3 & 6 & 2.7 \\
Clinical classification & & \\
III & 78 & 34.5 \\
IV & 148 & 65.5 \\
\hline
\end{tabular}

Follow-up. The patients were evaluated weekly during radiation therapy and were required to be followed-up by their attending radiation oncologist following the completion of their treatment, every 3 months for the first 2 years, every 6 months from the second through the fifth year and annually thereafter. Each follow-up included a complete examination, basic serum chemistry tests, chest X-ray and ultrasound of the liver and abdomen. Flexible fiberoptic endoscopy was performed at every visit after the treatment. MRI of the head and neck areas was performed every 6 months. Treatment-induced toxicities were assessed and scored according to the RTOG radiation morbidity scoring criteria at each follow-up.

Statistical analysis. The PFS and OS rates were calculated using the Kaplan-Meier method. The duration of time to local failure and distant metastasis was measured from the date of completion of the radiation therapy (including boost irradiation) until documented treatment failure. The OS duration was calculated from diagnosis until death or until the date of the last follow-up visit for the surviving patients. The statistical tests were performed using SPSS version 17.0. $\mathrm{P} \leq 0.05$ among the groups was considered to indicate a statistically significant difference.

\section{Results}

Patients and treatment evaluation. A total of 226 patients diagnosed with locally advanced NPC were included in this study. The patients received concurrent chemoradiotherapy (IMRT and TPF). The mean age of the patients was 43 years (range, 17-72). The majority of the patients were male (68.1\%) 
Table II. Kaplan-Meier estimate of progression-free survival (PFS) and overall survival (OS), according to pterygopalatine fossa, foramen rotundum, foramen ovale, foramen lacerum and cavernous sinus invasion, or lack thereof.

\begin{tabular}{|c|c|c|c|c|c|c|c|c|}
\hline Characteristic & No. & 5-year PFS (\%) & $\chi^{2}$ & P-value & No. & 5-year OS (\%) & $\chi^{2}$ & P-value \\
\hline \multicolumn{9}{|c|}{ Pterygopalatine fossa } \\
\hline+ & 120 & 43.3 & 11.74 & & 71 & 74.2 & 9.68 & \\
\hline- & 106 & 58.5 & & 0.001 & 155 & 89.6 & & 0.002 \\
\hline \multicolumn{9}{|c|}{ Foramen rotundum } \\
\hline+ & 38 & 7.9 & 47.73 & & 154 & 65.8 & 8.08 & \\
\hline- & 188 & 59.9 & & 0.000 & 72 & 84.6 & & 0.004 \\
\hline \multicolumn{9}{|l|}{ Foramen ovale } \\
\hline+ & 102 & 43.1 & 6.12 & & 78 & 74.5 & 5.11 & \\
\hline- & 124 & 53.5 & & 0.013 & 148 & 85.1 & & 0.024 \\
\hline \multicolumn{9}{|c|}{ Foramen lacerum } \\
\hline+ & 111 & 39.6 & 23.27 & & 36 & 77.5 & 2.10 & \\
\hline- & 115 & 60.9 & & 0.000 & 72 & 85.2 & & 0.148 \\
\hline \multicolumn{9}{|c|}{ Cavernous sinus } \\
\hline+ & 112 & 38.4 & 18.91 & & 78 & 77.7 & 1.92 & \\
\hline- & 114 & 62.3 & & 0.000 & 148 & 85.1 & & 0.166 \\
\hline
\end{tabular}

A

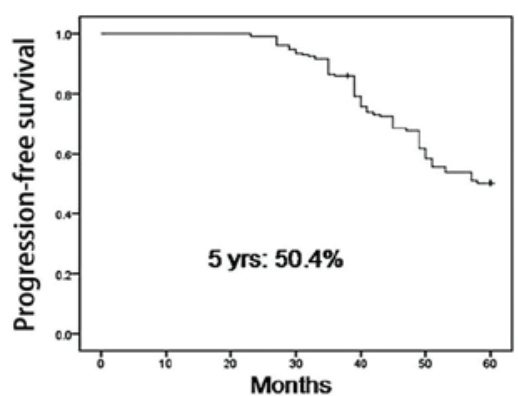

C

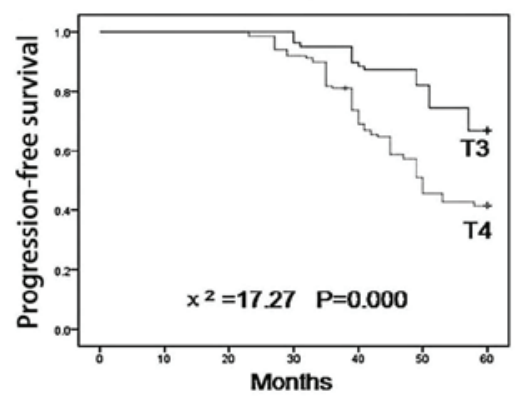

B

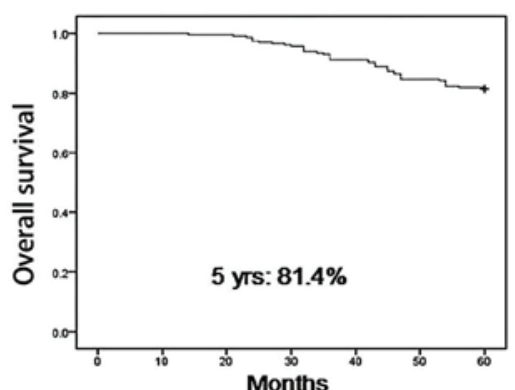

D

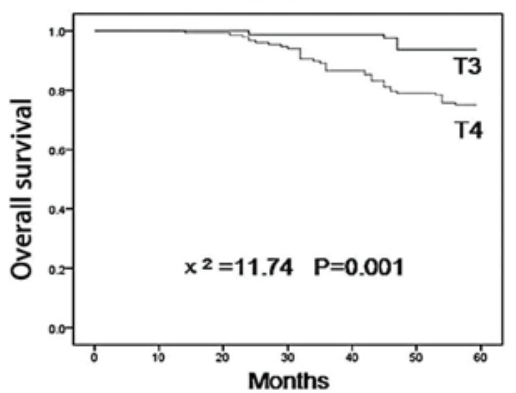

Figure 1. (A and B) Five-year progression-free survival and overall survival rates were 50.4 and $80.4 \%$, respectively. (C and D) Kaplan-Meier estimate of progression-free survival and overall survival, according to T-classification.

and Guangzhou stage III (34.5\%) and IV (65.5\%). The patients received 4-6 planned cycles of chemotherapy.

Both the primary site and neck nodes achieved an objective response rate of $98 \%$ for the response evaluation. The primary site showed an $87 \%$ complete response and the partial response was $11 \%$.

PFS and OS. With a median follow-up time of 35 months (range, 7-60), the 5-year OS rate was $81.4 \%$, with 93.6 and $75.0 \%$ for $\mathrm{T} 3$ and $\mathrm{T} 4$ lesions, respectively ( $\mathrm{P}=0.001$, Fig. 1). The 5-year PFS was 50.4\%, with 66.7 and $46.9 \%$ for T3 and
T4 lesions, respectively ( $\mathrm{P}<0.001$, Fig. 1). T-classification was a significant prognostic factor for PFS and OS. The subgroup analysis revealed that pterygopalatine fossa invasion was associated with a significantly lower 5-year PFS $(\mathrm{P}=0.001$, Table II) and $\mathrm{OS}(\mathrm{P}=0.002$, Table II), foramen rotundum invasion was associated with a significantly lower 5-year PFS $(\mathrm{P}<0.001)$ and OS $(\mathrm{P}=0.004)$, foramen ovale invasion was associated with a significantly lower 5-year PFS ( $\mathrm{P}=0.013$, Table II) and OS $(\mathrm{P}=0.024$, Table II) and foramen lacerum and cavernous sinus invasion were associated with a significantly lower 5-year PFS $(\mathrm{P}<0.001$ and $\mathrm{P}<0.001$, respectively; Table II $)$. By contrast, 
foramen lacerum and cavernous sinus invasion had no impact on the 5-year OS ( $\mathrm{P}=0.148$ and $\mathrm{P}=0.166$, respectively; Table II). None of the patients was lost during the follow-up period.

\section{Discussion}

NPC is considered unresectable due to its anatomical location and thus far radiation has been the conventional treatment approach $(6,7)$. Changing failure pattern has been noted in several publications and distant metastasis rates may be as high as $30 \%$, even with the integration of aggressive concurrent chemoradiotherapy schedules $(8,9)$.

Concurrent chemoradiation is an attractive approach to overcome the problem of distant metastases. However, it requires further investigation, since available post-experience data are sparse (10-13). China has reported the largest series of concurrent chemotherapy and IMRT data, with 323 locoregionally advanced NPC patients. The overall 3-year OS rate was $87.2 \%$. In the present study, the estimated 5-year PFS and OS rates were 50.4 and $80.4 \%$, respectively (14). Concurrent chemotherapy for locoregionally advanced NPC has been shown to be feasible and effective for local control, with high compliance. Although all our patients had locally advanced disease (stages III and IV), they exhibited excellent local control rates following concurrent chemotherapy or even salvage therapy.

The histopathological type and involvement of lymph nodes in the lower neck have been well-established as prognostic factors of NPC (15). In addition to these factors, tumor invasive range has been recognized as an important prognostic factor in the treatment of malignancy. Recently, tumor invasive range has been actively studied in head and neck malignancies. NPC is a tumor with a highly infiltrative growth pattern and a propensity to spread along the parapharyngeal space, as well as to the skull base and foramina (16). The invaded area may affect prognosis and may vary from extensive invasion, involving multiple sites, to only a small localized area, which, in some patients, may be the only site of extranasopharyngeal spread. The skull base foramina represent an unimpeded channel for tumor spread, although direct invasion of the bones bordering these foramina often occurs as well. The foramen ovale and foramen lacerum are the two most commonly involved foramina, which provide a route for tumor spread into the cranium. The inferior spread of the tumor, involving the hypoglossal nerve canal and jugular foramen, is less common. Orbital invasion usually occurs by direct tumor spread from the pterygopalatine fossa; the tumor may also spread directly to the cavernous sinus, leading to multiple cranial nerve palsies. This phenomenon suggests that NPC has an infiltrative growth pattern, often with a highly irregular tumor contour.

In this study, our results demonstrated that T-classification was a significant prognostic factor for PFS and OS. The subgroup analysis revealed that pterygopalatine fossa, foramen rotundum, foramen ovale, foramen lacerum and cavernous sinus invasion were all associated with the long-term results of concurrent chemotherapy for patients with NPC.

Other factors that contribute to the apparent tumor radioresistance must be considered. Although this study demonstrated an inverse correlation between tumor control and disease volume, several failures in small tumors and cures in cases with massive disease have been observed. These observations suggest that other factors, aside from those observed in this study, contribute to radiation response. Moreover, cellular factors, such as repopulation, intrinsic radioresistance, reoxygenation and redistribution, have been suggested as important variables for tumor control (17).

In conclusion, volumetric analysis of the primary tumor and lymph nodes, anatomical sites involved and intracranial extension should be included in the current TNM staging system for NPC, in order to optimize the staging system.

\section{References}

1. Perri F, Bosso D, Buonerba C, et al: Locally advanced nasopharyngeal carcinoma: current and emerging treatment strategies. World J Clin Oncol 2: 377-383, 2011.

2. Al-Sarraf M and Reddy MS: Nasopharyngeal carcinoma. Curr Treat Options Oncol 3: 21-32, 2002.

3. Yi JL, Gao L, Xu GZ, et al: Treatment results of intensitymodulated radiotherapy for nasophyngeal carcinoma: an analysis of 147 patients. Chin J Radiat Oncol 17: 329-334, 2008.

4. Al-Sarraf M, LeBlanc M, Giri PG, et al: Chemoradiotherapy versus radiotherapy in patients with advanced nasopharyngeal cancer: phase III randomized Intergroup study 0099. J Clin Oncol 16: 1310-1317, 1998.

5. Baujat B, Audry H, Bourhis J, et al: Chemotherapy in locally advanced nasopharyngeal carcinoma: an individual patient data meta-analysis of eight randomized trials and 1753 patients. Int $\mathrm{J}$ Radiat Oncol Biol Phys 1: 47-56, 2006.

6. Ishiki $\mathrm{H}$ and Tahara $\mathrm{M}$ : Induction chemotherapy followed by chemoradiotherapy for the patients with far-advanced nasopharyngeal carcinoma - our treatment strategy. Gan To Kagaku Ryoho 39: 698-701, 2012 (In Japanese).

7. Caponigro F, Longo F, Ionna F, et al: Treatment approaches to nasopharyngeal carcinoma: a review. Anticancer Drugs 21: 471-477, 2010.

8. Heng DMK, Wee J, Fong KW, et al: Prognostic factors in 677 patients in Singapore with nondisseminated nasopharyngeal carcinoma. Cancer 86: 1912-1920, 1999.

9. Ma J, Mai HQ, Hong MH, et al: Is the 1997 AJCC staging system for nasopharyngeal carcinoma prognostically useful for Chinese patient population? Int J Radiat Oncol Biol Phys 50: 1181-1189, 2001.

10. Zhang L, Zhao C, Peng PJ, et al: Phase III study comparing standard radiotherapy with or without weekly oxaliplatin in treatment of locoregionally advanced nasopharyngeal carcinoma: preliminary results. J Clin Oncol 23: 8461-8468, 2005.

11. Lee AW, Lau WH, Tung SY, et al: Preliminary results of a randomized study on therapeutic gain by concurrent chemotherapy for regionally-advanced nasopharyngeal carcinoma: NPC-9901 Trial by the Hong Kong Nasopharyngeal Cancer Study Group. J Clin Oncol 23: 6966-6975, 2005.

12. Wee J, Tan EH, Tai BC, et al: Randomized trial of radiotherapy versus concurrent chemoradiotherapy followed by adjuvant chemotherapy in patients with American Joint Committee on Cancer/International Union against cancer stage III and IV nasopharyngeal cancer of the endemic variety. J Clin Oncol 23: 6730-6738, 2005.

13. Lu H, Peng L, Yuan X, et al: Concurrent chemoradiotherapy in locally advanced nasopharyngeal carcinoma: a treatment paradigm also applicable to patients in Southeast Asia. Cancer Treat Rev 35: 345-353, 2009.

14. Lin S, Pan J, Han L, et al: Nasopharyngeal carcinoma treated with reduced-volume intensity-modulated radiation therapy: report on the 3-year outcome of a prospective series. Int J Radiat Oncol Biol Phys 75: 1071-1078, 2009.

15. Han L, Lin SJ, Pan JJ, et al: Prognostic factors of 305 nasopharyngeal carcinoma patients treated with intensity-modulated radiotherapy. Chin J Cancer 29: 145-150, 2010.

16. Lu CH, Yang CY, Wang CP, et al: Imaging of nasopharyngeal inflammatory pseudotumours: differential from nasopharyngeal carcinoma. Br J Radiol 83: 8-16, 2010.

17. Oehler C, Dickinson DJ, Broggini-Tenzer A, et al: Current concepts for the combined treatment modality of ionizing radiation with anticancer agents. Curr Pharm Des 13: 519-535, 2007. 